\title{
Cardiac magnetic resonance radiofrequency tissue tagging for diagnosis of constrictive pericarditis: A proof of concept study
}

\author{
John A. Power, BS, ${ }^{\text {a,b }}$ Diane V. Thompson, MS, ${ }^{\text {a }}$ Geetha Rayarao, MS, ${ }^{a}$ Mark Doyle, PhD, ${ }^{a}$ and \\ Robert W. W. Biederman, MD, FACC, FAHA ${ }^{\mathrm{a}}$
}

\begin{abstract}
Objective: Invasive cardiac catheterization is the venerable "gold standard" for diagnosing constrictive pericarditis. However, its sensitivity and specificity vary dramatically from center to center. Given the ability to unequivocally define segments of the pericardium with the heart via radiofrequency tissue tagging, we hypothesize that cardiac magnetic resonance has the capability to be the new gold standard.
\end{abstract}

Methods: All patients who were referred for cardiac magnetic resonance evaluation of constrictive pericarditis underwent cardiac magnetic resonance radiofrequency tissue tagging to define visceral-parietal pericardial adherence to determine constriction. This was then compared with intraoperative surgical findings. Likewise, all preoperative cardiac catheterization testing was reviewed in a blinded manner.

Results: A total of 120 patients were referred for clinical suspicion of constrictive pericarditis. Thirty-nine patients were defined as constrictive pericarditis positive solely via radiofrequency tissue-tagging cardiac magnetic resonance, of whom 21 were positive, 4 were negative, and 1 was equivocal for constrictive pericarditis, as defined by cardiac catheterization. Of these patients, 16 underwent pericardiectomy and were surgically confirmed. There was $100 \%$ agreement between cardiac magnetic resonance-defined constrictive pericarditis positivity and postsurgical findings. No patients were misclassified by cardiac magnetic resonance. In regard to the remaining constrictive pericarditis-positive patients defined by cardiac magnetic resonance, 10 were treated medically, declined, were ineligible for surgery, or were lost to follow-up. Long-term follow-up of those who were constrictive pericarditis negative by cardiac magnetic resonance showed no early or late crossover to the surgery arm.

Conclusions: Cardiac magnetic resonance via radiofrequency tissue tagging offers a unique, efficient, and effective manner of defining clinically and surgically relevant constrictive pericarditis. Specifically, no patient who was identified with constriction via cardiac magnetic resonance underwent inappropriate sternotomy. However, catheterization had substantial and unacceptable false-positive and false-negative rates with important clinical ramifications. (J Thorac Cardiovasc Surg 2016;151:1348-55)

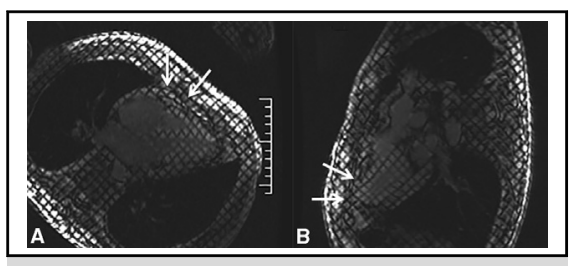

Magnetic resonance images depicting an example of tissue tagging to delineate the pericardium and myocardium.

\section{Central Message}

We present cardiac magnetic resonance imaging tissue tagging to diagnose constrictive pericarditis. Its accuracy and correlation with surgically confirmed pericardiectomy are $100 \%$.

\section{Perspective}

Constrictive pericarditis is a difficult disease to diagnose. We present the utility of cardiac magnetic resonance imaging, a noninvasive technology, via radiofrequency tissue tagging diagnose constrictive pericarditis. Its accuracy and correlation with surgically confirmed pericardiectomy are $100 \%$. This approach is viable on all manufacturers' magnetic resonance imaging scanners and is easier, cheaper, safer, and more robust than current modalities.

See Editorial Commentary page 1356.

\footnotetext{
From the ${ }^{a}$ Department of Medicine Cardiovascular Magnetic Resonance Center, Allegheny General Hospital, Pittsburgh, Pa; and ${ }^{\mathrm{b}}$ University of Connecticut School of Dental Medicine, Farmington, Conn.

Funding was internal.

Received for publication July 22, 2015; revisions received Dec 4, 2015; accepted for publication Dec 16, 2015; available ahead of print Jan 24, 2016.

Address for reprints: Robert W. W. Biederman, MD, FACC, FAHA, Cardiovascular Magnetic Resonance Imaging, Temple University School of Medicine, Carnegie Mellon University, Allegheny Health Network, Allegheny General Hospital, 320 E North Ave, Pittsburgh, PA 15212 (E-mail: Rbiederm@wpahs.org).

$0022-5223 / \$ 36.00$

Copyright (c) 2016 by The American Association for Thoracic Surgery

http://dx.doi.org/10.1016/j.jtcvs.2015.12.035
}

Constrictive pericarditis (CP) is encountered increasingly in clinical practice and may present with classic symptoms or, more frequently in today's environment, innocuously

Scanning this QR code will take you to the article title page.

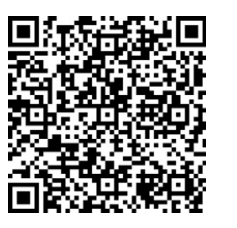




\section{Abbreviations and Acronyms \\ $\mathrm{CI}=$ confidence interval \\ $\mathrm{CMR}=$ cardiac magnetic resonance \\ $\mathrm{CP}=$ constrictive pericarditis \\ $\mathrm{CT}=$ computed tomography \\ MRI = magnetic resonance imaging \\ $\mathrm{RF}=$ radiofrequency \\ $\mathrm{SSFP}=$ steady-state free precession}

masquerade as another disorder. $\mathrm{CP}$ is elusive. The diagnosis often is unheralded and escapes diagnosis for months and even years, subjecting the patient to a progressive burden that, but for a diagnosis, could be treated surgically relatively easily. However, too often the index of suspicion is low. When coupled with inexact noninvasive and invasive tools to unequivocally make the diagnosis, a vicious circle ensues. Thus, this study seeks to present a straightforward manner in which to unequivocally make a clinical diagnosis of $\mathrm{CP}$ and to demonstrate the utility of such an approach in the surgical suite with confirmation of the diagnosis. To be clear, many approaches have been advocated in the past, chiefly reliant on echocardiography, computed tomography (CT), or catheterization. Few have used the emerging new tool of cardiac magnetic resonance (CMR). In the last decade, CMR unfailingly has been demonstrated to be the "gold standard" in a host of cardiovascular disease entities, such as biventricular function, viability, cardiomyopathies, and congenital heart disease, and, more recently, in post-heart transplant recipients. The unique capability to integrate function and physiology not limited by field of view, spatial/temporal resolution, or obligate need for invasive strategy, radioisotopes, or radiation has natural attributes serving to underpin the likelihood for success in this arena. Thus, it would not be unexpected that elucidation of yet another complex pathophysiologic process could be possible via CMR. This study establishes the utility of CMR in CP and provides the surgical basis for its place in the armamentarium of tools available to the physician and particularly to the discerning surgeon facing the consternation of $\mathrm{CP}$.

In brief, the current study defines the anatomic and physiologic issues and quickly reminds the reader of the multitude of imaging and invasive tools currently available to the surgeon for $\mathrm{CP}$ while setting the stage for the focus of the CMR CP study.

\section{PERICARDIAL ANATOMY}

The pericardium consists of 2 layers: the parietal and visceral layers. In between these layers is 5 to $20 \mathrm{~mL}$ of pericardial fluid. The pericardial fluid allows the 2 membranes to slide freely with reduced friction. ${ }^{1} \mathrm{CP}$ is the fibrosis and calcification of the pericardium, which over time prevents the heart from filling during diastole. ${ }^{2}$ A multitude of diseases can cause irritation of the pericardium, in turn leading to $\mathrm{CP} .{ }^{1}$ In the past, a major cause of $\mathrm{CP}$ was tuberculosis. ${ }^{3}$ With the decline of tuberculosis cases in the United States, the most frequent current cause of $\mathrm{CP}$ in the 21 st century is cardiac surgery and radiation therapy. ${ }^{1,3}$

\section{CLINICAL CONUNDRUM}

$\mathrm{CP}$ is a problematic disease to diagnose. ${ }^{4}$ One of the key identifiers in diagnosing constriction is a thickened pericardium. However, it has been shown in contemporary studies that only $80 \%$ of patients with surgically proven $\mathrm{CP}$ have thickened pericardium $(>4 \mathrm{~mm}) .{ }^{5}$ This problem has only intensified as effusive constriction because tuberculosis is seen less often, whereas postpericardiotomy due to surgical intervention is increasingly responsible for contemporary $\mathrm{CP}$. Another major problem with diagnosing $\mathrm{CP}$ is that it can mimic diseases as divergent as myocardial infarction and cirrhosis. This is a vexing clinical problem because failure to diagnose accurately leads to delays in correct therapy or inappropriate pericardial stripping.

\section{COMMON TOOLS AVAILABLE FOR DIAGNOSING CONSTRICTIVE PERICARDITIS \\ Cardiac Catheterization}

Historically, most patients presenting with $\mathrm{CP}$ have been diagnosed via cardiac catheterization. Cardiac catheterization has been considered the de facto "gold standard" for the evaluation and confirmation of $\mathrm{CP}^{2}$ Cardiac catheterization has the ability to determine the equalization of diastolic pressures in the right and left ventricles, the square root sign, and the rapid $\mathrm{x}$ and $\mathrm{y}$ descents in the atrial pressure curves. However, these signs also can be seen in patients presenting with restrictive disease. $^{2,5}$ Ventricular interdependence can be used to differentiate between constriction and restriction, simultaneously using the left ventricular and right ventricular pressures. Patients with constriction will have a decreased wedge pressure during the first beat of inspiration, whereas patients with restrictive disease will not demonstrate a decrease in wedge pressure. ${ }^{2,5} \mathrm{~A}$ negative cardiac catheterization is due to the lack of equilibration of pressures (left ventricular end-diastolic pressure, right ventricular end-diastolic pressure, left atrium, and right atrium), which is determined by the lack of a dip and plateau, also known as a "square root sign." However, cardiac catheterization is invasive and requires a significant amount of training to be proficient. Its sensitivity and specificity are improved only by evaluating cumbersome ventricular interdependence requiring multiple hemodynamic catheters, which, in our experience, is well done in limited laboratories. 


\section{Computed Tomography}

$\mathrm{X}$-ray CT is the use of 2-dimensional $\mathrm{x}$-ray images to generate a 3-dimensional image. CT helps diagnose CP through direct visualization of the pericardium. CT can be used to identify thickened pericardium more readily than an echocardiogram. However, the problem exists that in the 21 st century, only $80 \%$ of patients with confirmed CP have thickened pericardium. ${ }^{5}$ One of the other primary identifiers of $\mathrm{CP}$ when using $\mathrm{CT}$ is pericardial calcification. However, only $20 \%$ of patients with $\mathrm{CP}$ present with calcification. ${ }^{5}$ In addition, patients are exposed to intravenously administered iodinated contrast material and ionizing radiation.

\section{Echocardiography}

Echocardiography uses ultrasound techniques to produce 2-dimensional images of the heart. Echocardiography has been used to diagnose CP. This method is not reliable enough to produce a diagnosis because there are fewer patients with $\mathrm{CP}$ presenting with a thickened pericardium. ${ }^{6}$ Also, the echocardiogram has less than optimal visual sensitivity and frequently can be subject to technical limitations, especially in patients with chronic lung disease. ${ }^{2}$

\section{Cardiac Magnetic Resonance Imaging}

When clinical suspicion arises, a technique that is able to comprehensively and accurately define anatomic or physiologic $\mathrm{CP}$ would be of obvious value. Cardiac magnetic resonance imaging (MRI) is a noninvasive procedure that determines the function and structure of the cardiovascular system. Noninvasive cardiac MRI could have a potential role to be equivalent or even superior, given its ability to detect both the anatomic and physiologic impact of an abnormal pericardium. CMR can define a thickened pericardium through steady-state free precession (SSFP) imaging and double-inversion recovery sequences. ${ }^{7}$ More recently, radiofrequency (RF) tissue tagging has been suggested for possessing certain morphologic characteristics that naturally lend themselves toward distinction of visceral-partial attributes. ${ }^{8}$ Namely, CMR uses RF tissue tagging to facilitate identification of patients positive for $\mathrm{CP}$ by defining visceral-parietal adherence patterns. RF tissue tagging is an imaging technique that can image motion via spatially modulating the degree of magnetization before imaging after the intrepid initial observation of Axel and Dougherty. ${ }^{9}$ Another advantage of CMR is that this imaging modality is less operator dependent than an echocardiogram or a cardiac catheterization. Further, the underpinning of the full mechanism of the "septal bounce" so often used in echocardiography was elucidated this year, providing greater insight into the physiology of CP via CMR. ${ }^{10}$
In this study, CMR was restricted to the standard imaging planes rather than the free range of imaging planes with echocardiograms. ${ }^{1}$ Therefore, we hypothesize that CMR may be a contender for a new noninvasive standard for the evaluation of $\mathrm{CP}$, an important clinical conundrum. We further hypothesize that CMR via SSFP and RF tissue tagging can define visceral-parietal adherence and achieve a high degree of accuracy as confirmed at cardiac surgery.

\section{MATERIALS AND METHODS}

An expedited institutional review board-approved retrospective chart review was conducted on patients at Allegheny General Hospital's Cardiovascular MRI suite from January 2000 to August 2013. The records of all patients referred for or diagnosed with $\mathrm{CP}$ were examined. These patients were split into 2 groups according to CMR results: CP positive and $\mathrm{CP}$ negative. A positive diagnosis of $\mathrm{CP}$ via MRI was made only if the patient had visceral-parietal adherence as defined by RF tissuetagging interrogation; likewise, negative $\mathrm{CP}$ was the inverse. CMR was performed and interpreted independently of the clinical presentation.

\section{Radiofrequency Tissue Tagging}

A General Electric (Milwaukee, Wis) 1.5T CVI CMR was used for all imaging. Blood pressure, heart rate, and oximetry monitoring were performed, and, if necessary, hemodynamic monitoring was performed for ventilated patients. Vector electrocardiogram gating was used for the critical recognition of the R-wave necessary for dependent myocardial tagging grid pattern. RF tags typically are deployed to analyze myocardial contraction during systole. Orthogonal tags lines are created on detection of the QRS complex of the electrocardiogram. The resulting tags then track myocardial motion during the cardiac cycle, thus reflecting the underlying myocardial deformation. However, in this application, the focus of the saturation grid was on the ancillary pericardial surfaces. By using the GE cardiac-triggered CMR system with a $40 \mathrm{mT} / \mathrm{m}$ maximum gradient strength and $150 \mathrm{mT} / \mathrm{m} / \mathrm{ms}$ maximum slew rate, scout images were obtained to plan double-oblique views in the vertical long-axis and horizontal long-axis chamber views from which short-axis contiguous 8-mm slices traversing the mitral valve plane through the left ventricular apex were acquired. A cine RF intramyocardial tissue tagging was performed using a cine sequence (SSFP [Steady State Free Precession]; General Electric) incorporating an 8-mm slice with deposition of an orthogonal grid pattern and an intratag stripe distance of $8 \mathrm{~mm}$. Parameters were field of view of $38 \mathrm{~cm}^{2}$, matrix of $256 \times 192$, and flip angle of $45^{\circ}$. The temporal resolution was $30 \pm 3 \mathrm{~ms}$, repetition time of $3.7 \mathrm{~ms}$, echocardiography time of $2.2 \mathrm{~ms}, 100 \%$ phase field of view, and 0.75 number of excitations. Standard visual interpretation was used for analysis by a physician (RWWB) with more than 15 years of experience with CMR-defined CP and RF myocardial deformational analysis.

\section{Definition of Constriction via Cardiac Magnetic Resonance}

Visceral-pericardial adherence via CMR was defined as the failure for orthogonally aligned RF tagged stripes to "slide" past each other along the pericardium. Slippage between the visceral and parietal pericardium is seen in normal individuals, and the absence of such defines those with anatomic CP. Corresponding physiologically important constriction was present if other mitigating factors were present, including an intermittent septal bounce caused by visceral-parietal adherence, evidence of only focal adherence, or dilated inferior vena cava, superior vena cava, and coronary sinus but was not necessary for the RF-defined CP diagnosis in this study. Also, real-time SSFP with deep inspiration imaging was used for confirmation for 2 cases (but not used routinely). Of note, the definition 
of CP was not dependent on the anatomic thickness of the pericardium. In some cases, there was no observed thickening, ventricular interdependence, or square root sign. To reflect the impact and veracity of the RF tissue tagging alone to diagnose $\mathrm{CP}$, the study by intent was restricted for the research component. In all cases, the findings of the tissue tagging were in concordance with the traditional CMR (and echocardiography) finding of septal bounce, dilated superior vena cava/inferior vena cava, and interventricular dependence physiology. Thus, for the purpose of this report, the definition of CP was made solely by the presence of "lack of slippage" so defined by RF-tissue tagging. All patient records were examined for corresponding temporally related echocardiograms, CT scans, cardiac catheterization, and pericardial surgeries to independently corroborate the CMR findings.

\section{Statistics}

Categoric variables were presented as counts and frequencies. The $95 \%$ confidence intervals (CIs) for proportions were calculated according to the method of Wilson. ${ }^{11}$ Normality was defined by the Kolmogorov-Smirnov test. Sensitivity, specificity, and prevalence were calculated with $95 \%$ CIs. Data were analyzed using SAS version 9.2 (SAS Institute Inc, Cary, NC).

\section{RESULTS}

A total of 120 patients were referred for CMR at Allegheny General Hospital, Pittsburgh, Pennsylvania, for CP from January 2000 to August 2012. Patients underwent evaluation for $\mathrm{CP}$ by $\mathrm{CMR}$ on request of the referring physician for predominant pathophysiology involving chronic, recurrent pericarditis, less so postsurgical and even less so tuberculosis. No case of acute pericarditis was referred or went to surgery. The average scan time was 45 to 60 minutes; no complications were encountered during the examination. Thirty-nine (33\%) of those patients were diagnosed as CP positive according to CMR, and 81 $(68 \%)$ of those patients were diagnosed as CP negative. Twenty-six $(67 \%)$ of the $39 \mathrm{CMR} \mathrm{CP}+$ patients underwent cardiac catheterizations; 21 (81\%) were positive, $4(15 \%)$ were negative, and $1(4 \%)$ was equivocal. Twenty-seven $(69 \%)$ of the 39 aforementioned CMR-CP-positive patients went on to further treatment; $16(59 \%)$ were referred and went to surgery for pericardiectomy, $10(37 \%)$ were treated medically by intent, and $1(4 \%)$ declined treatment (Figure 1, A). Twelve CP-positive patients did not undergo surgery because the clinician decided not to send the patient to surgery. ${ }^{11}$ Only 1 patient was lost because of follow-up.

The cardiothoracic surgery reports were reviewed and demonstrated $100 \%$ agreement between CMR and surgical findings; all $16 \mathrm{CMR}$ CP-positive patients were confirmed at surgery and underwent pericardial stripping. A total of 81 patients were $\mathrm{CP}$ negative as demonstrated by CMR. Twenty-four $(30 \%)$ of those patients had cardiac catheterizations, $18(75 \%)$ were negative, $6(25 \%)$ were positive, and $1(4 \%)$ was equivocal (Figure 1, B). Likewise, the echocardiography reports were reviewed. Not all patients underwent echocardiography; however, 7 of 12 were shown to be $\mathrm{CP}+$ via $\mathrm{CMR}$, and 67 of 81 were defined as negative (or no comment) via echocardiography who

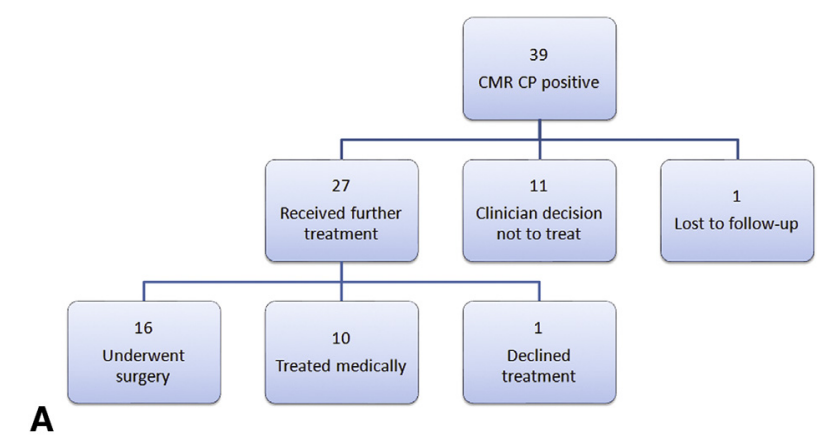

A

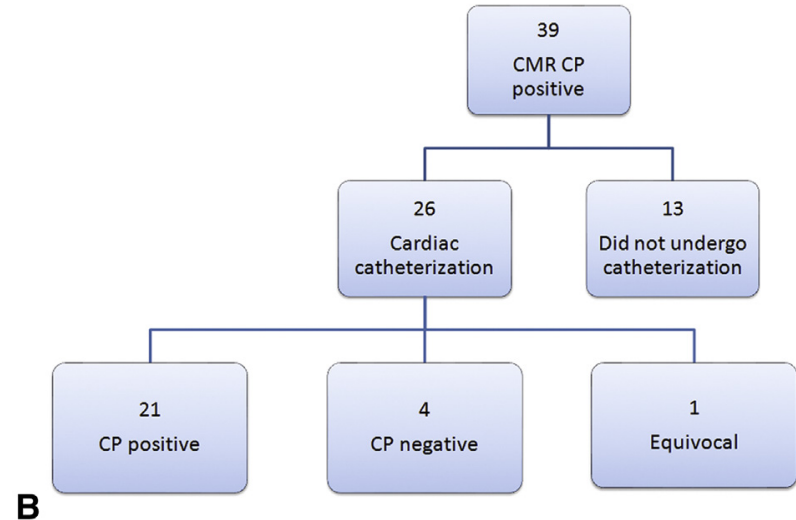

FIGURE 1. A, Treatment after $\mathrm{CMR} \mathrm{CP}+$. B, Catheterization laboratory use in $\mathrm{CMR} \mathrm{CP}+. C M R$, Cardiac magnetic resonance; $C P$, constrictive pericarditis.

were classified as $\mathrm{CP}-$ via CMR. Finally, 2 patients $(3 \%)$ who were positive according to cardiac catheterization but negative via CMR underwent surgery, but both patients were found to be negative for $\mathrm{CP}$ and did not undergo pericardial stripping (Table 1). All $16 \mathrm{CP}$-positive patients had not undergone prior cardiothoracic procedures. One of the 16 patients was diagnosed specifically with localized $\mathrm{CP}$, and 1 patient was diagnosed specifically with effuse $\mathrm{CP}$.

Cardiac MRI was able to diagnose patients with a sensitivity and specificity of $100 \%$ when compared with the surgical results. A surgical positive result is defined by the surgeon using the totality of adherence, extent, and atrial relief criteria, as well as documentation of a decrease in atrial pressure. Although the specificity of the trial was

TABLE 1. Summary of patients diagnosed by different modalities compared with those diagnosed by cardiac magnetic resonance

\begin{tabular}{lcc}
\hline & CMR CP & CMR CP- \\
& $(\mathbf{3 7})$ & $\mathbf{( 8 1 )}$ \\
\hline Cardiac catheterization CP+ & $21 / 26(81 \%)$ & $6 / 24(25 \%)$ \\
Surgery CP+ & $16 / 16(100 \%)^{*}$ & 0 \\
Echocardiogram CP+ & $7 / 12(58 \%)$ & $67 / 81(83 \%)$ \\
Cardiac catheterization CP- & $4 / 26(15 \%)$ & $18 / 24(75 \%)$ \\
Surgery CP- & $0(0 \%)$ & $2 / 2(100 \%)$ \\
\hline
\end{tabular}

$C M R$, Cardiac magnetic resonance; $C P$, constrictive pericarditis. $*$ Of the 39 patients, 16 had surgery; of the remaining, 10 were treated medically, 1 declined treatment, and 1 was lost to follow-up. 
TABLE 2. Summary of patients diagnosed by cardiac magnetic resonance versus the surgical results

\begin{tabular}{lccr}
\hline & $\begin{array}{c}\text { Positive surgery } \\
\text { constriction }\end{array}$ & $\begin{array}{c}\text { Negative surgery } \\
\text { constriction }\end{array}$ & Total \\
\hline Abnormal - CMR & 16 & 0 & 16 \\
Normal - CMR & 0 & 2 & 2 \\
Total & 16 & 2 & 18 \\
\hline
\end{tabular}

$C M R$, Cardiac magnetic resonance.

$100 \%$, the CI is necessarily large because of the relatively small number of patients (Table 2-4). Of note, of the 120 patients who were referred to CMR for CP, $52(43 \%)$ had cardiac catheterization before or after the MRI. Twenty-seven $(53 \%)$ of the cardiac catheterizations indicated that the patient had CP. Twenty-five $(48 \%)$ of the cardiac catheterizations were normal, indicating that the patient was negative for CP (Table 3). Volume loading of $500 \mathrm{~mL}$ was routinely performed if $\mathrm{CP}$ was suspected. Of the 16 patients positive for $\mathrm{CP}$ according to $\mathrm{CMR}$ and surgery, 15 (94\%) had cardiac catheterizations (Table 2). Fourteen patients $(93 \%)$ were positive for $\mathrm{CP}$, and 1 patient $(7 \%)$ was negative for $\mathrm{CP}$. Of the 81 patients negative for constriction according to cardiac MRI, 2 cardiac catheterizations were positive. Both of these patients went to surgery. The results of both surgeries were negative for CP. During the subsequent surgery, an intrapericardial mass was found in 1 of the patients as predicted by CMR (Table 2). Follow-up was complete at $18 \pm 4$ months, documenting in the CP-negative cohort the absence of crossover into a surgical arm.

\section{DISCUSSION}

$\mathrm{RF}$ tagging has emerged relatively recently as a way to diagnose CP as shown in an index 1999 case report. ${ }^{12} \mathrm{~A}$ single small study done in Morocco showed that CMR, which included sparse RF imaging techniques, had an overall sensitivity of $88 \%$, a specificity of $100 \%$, and an accuracy of $93 \% .{ }^{13}$ The chief clinical impediment with diagnosing $\mathrm{CP}$ is differentiating $\mathrm{CP}$ from restrictive cardiomyopathy. ${ }^{14}$ However, once $\mathrm{CP}$ is suspected, robust modalities to accurately define $\mathrm{CP}$ are limited. The penalty for overdiagnosis is inadvertent open sternotomy with its obligate morbidity/mortality. Likewise, underdiagnosing thwarts lifesaving surgical intervention. Because of the

TABLE 3. Summary of patients diagnosed by cardiac catheterization versus the surgical results

\begin{tabular}{lccr}
\hline & $\begin{array}{c}\text { Positive surgery } \\
\text { constriction }\end{array}$ & $\begin{array}{c}\text { Negative surgery } \\
\text { constriction }\end{array}$ & Total \\
\hline Abnormal - catheterization & 14 & 1 & 15 \\
Normal - catheterization & 2 & 0 & 2 \\
Total & 16 & 1 & 17 \\
\hline
\end{tabular}

TABLE 4. Comparing cardiac magnetic resonance versus surgery results with cardiac catheterization versus surgery results

\begin{tabular}{lcc}
\hline & CMR & Catheterization \\
\hline Sensitivity & $16 / 16: 1(100 \%)$ & $14 / 16: 0.875(88 \%)$ \\
& $95 \% \mathrm{CI}, 0.76-1.0$ & $95 \% \mathrm{CI}, 0.66-0.99$ \\
Specificity & $2 / 2: 1(100 \%)$ & $0 / 1: 0.0(0 \%)$ \\
& $95 \% \mathrm{CI}, 0.20-1.0$ & $95 \% \mathrm{CI}, 0.0-0.80$ \\
Accuracy & $1(100 \%)$ & $21 / 26(81 \%)$ \\
Positive predictive value & $16 / 16: 1(100 \%)$ & $14 / 15: 0.933(93 \%)$ \\
& $95 \% \mathrm{CI}, 0.76-1.0$ & $95 \% \mathrm{CI}, 0.60-0.98$ \\
Negative predictive value & $2 / 2: 1(100 \%)$ & $0 / 2: 0.0(0 \%)$ \\
& $95 \% \mathrm{CI}, 0.20-1.0$ & $95 \% \mathrm{CI}, 0.0-0.95$ \\
\hline
\end{tabular}

$C M R$, Cardiac magnetic resonance; $C I$, confidence interval.

limited ability of conventional approaches to diagnose $\mathrm{CP}$, we believe failure to correctly diagnose $\mathrm{CP}$ leads to substantial medical errors, most of which we propose can be negated by judicious use of CMR. ${ }^{8}$

CMR tagging may offer a novel, accurate means of diagnosing constriction unlike other diagnostic modalities. ${ }^{15}$ In appropriate cases, CMR can be used in patients with pacemakers to make the $\mathrm{CP}$ diagnosis (Figure 2). ${ }^{8,15,16}$ The sensitivity and specificity of cardiac catheterization were not as accurate as for cardiac MRI. However, the determination of cardiac catheterization's specificity was limited because of the obvious inability to confirm CP-negative patients via surgery. It is important to note that in the past, cardiac catheterization has been deemed the most accurate way to diagnose CP. However, the principle finding of this study was that cardiac MRI is capable of diagnosing a patient who is positive for $\mathrm{CP}$ with $100 \%$ sensitivity and accuracy as confirmed independently by CT surgery in our study. This was a greater sensitivity than cardiac catheterization in diagnosing CP (100\% vs $88 \%)$ (Table 4). This was achieved through a novel use of CMR's ability to noninvasively track visceral-parietal pericardial interactions through RF tissue tagging. CMR defined the presence of visceral-parietal adherence (absence of slippage) as pathognomonic for the diagnosis of CP. The RF tissue tagging technique is able to delineate this hallmark feature of CP in a manner superior to echocardiography and CT (Figures 3 and 4). With CMR's ability to discover or confirm CP with $100 \%$ sensitivity and accuracy, we propose that CMR can be the primary diagnostic tool to diagnose CP. CMR also can be used as a safety net to confirm other less accurate diagnostic tools for the diagnosis of $\mathrm{CP}$, thus preventing patients from undergoing a procedure with a high morbidity. ${ }^{17}$

This is an easily learned skill, but it does require attention to detail, which is well in the purview of radiologists and cardiologists. The technique is used clinically and easily learned to include the sensitivity and specificity. 


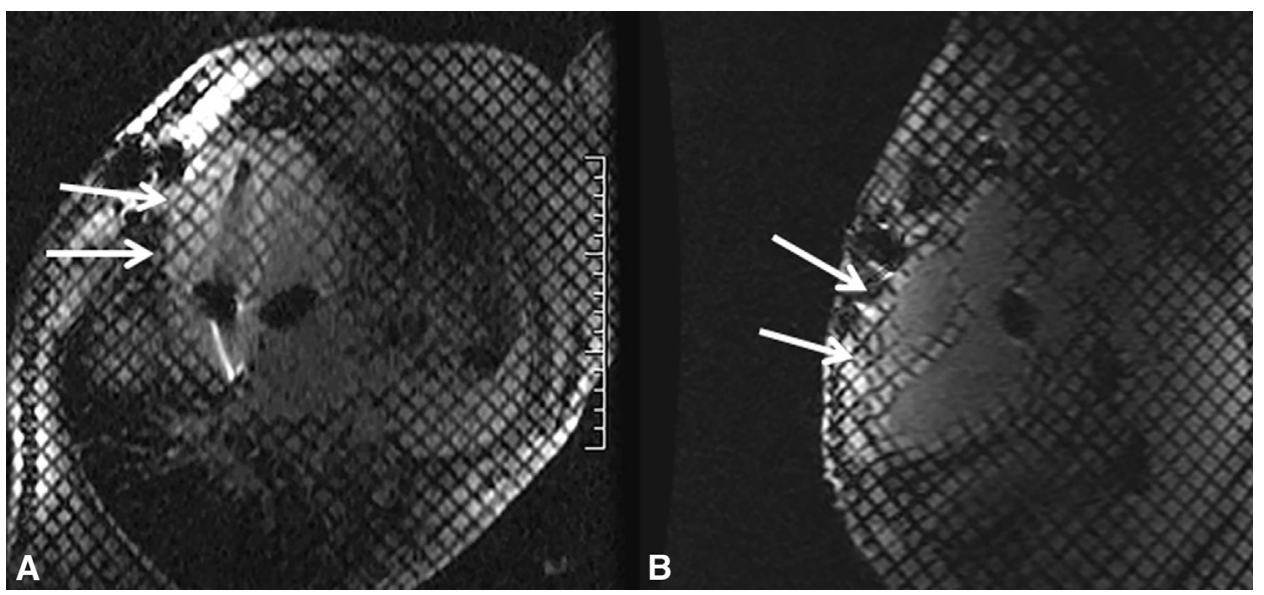

FIGURE 2. A and B, RF tissue tagging during ventricular systole in a 46-year-old woman with 15 months of indolent but progressive debilitating symptoms despite 3 echocardiograms, $1 \mathrm{CT}$, and 2 cardiac catheterizations ( 1 with $0.5 \mathrm{~L} \mathrm{NaCL}$ volume loading) yet only equivocal findings at best for CP. CMR was performed in less than 8 minutes (because of the indwelling dual-chamber pacemaker) quickly and unequivocally confirming extensive constrictive physiology. All areas in this case, including the atria, illustrate visceral-parietal adherence consistent with CP (arrows), which was easily confirmed 1 hour later in the surgical suite. A, The pacemaker in the right ventricle with minimal artifact (and s/p MVR and TV annuloplasty). ${ }^{14}$

\section{Clinical Perspective}

Does CMR tagging require sophisticated sequences or training and is it expensive? These are frequent questions we encounter. The answer is "no" for both. The RF tissue tagging sequence is part of all cardiac packages. A center that can image the heart simply chooses "tagging" on the scan prescriptions, and the tags are seamlessly added to a standard gradient recalled echocardiography sequence (they are not part of an SSFP sequence). It should be noted that it does not require a dedicated cardiac MRI scanner or particular local cardiac expertise; indeed, the first RF myocardial tagging was perform by Axel and Dougherty ${ }^{9}$ in Philadelphia in a radiology MRI center. A CMR examination is far less expensive than a cardiac catheterization in terms of both dollars and obligate morbidity. The scan takes approximately 30 minutes inclusive of baseline imaging. No contrast is explicitly required, although we and others recently showed that gadolinium via the late gadolinium enhancement sequence can provide insight into the acuity of the pericardial thickening with therapeutic ramifications. $^{18}$

\section{Study Limitations}

Dedicated CMR capabilities are not ubiquitous, although RF tagging is available on every vendor's CMR platform since 2001. The interpretative skills for CP are a learned technique but can be mastered within the confines of a Cardiology or Radiology fellowship training or case review. ${ }^{8}$ The assessment essentially is visual at present but may become automated in future analysis packages. A tagging grid line spacing of $7 \mathrm{~mm}$ was used, which permits tracking to 2-mm-thick pericardium. However, pericardium that is thinner than this, typically not a clinical likelihood, may not be represented adequately in tagged

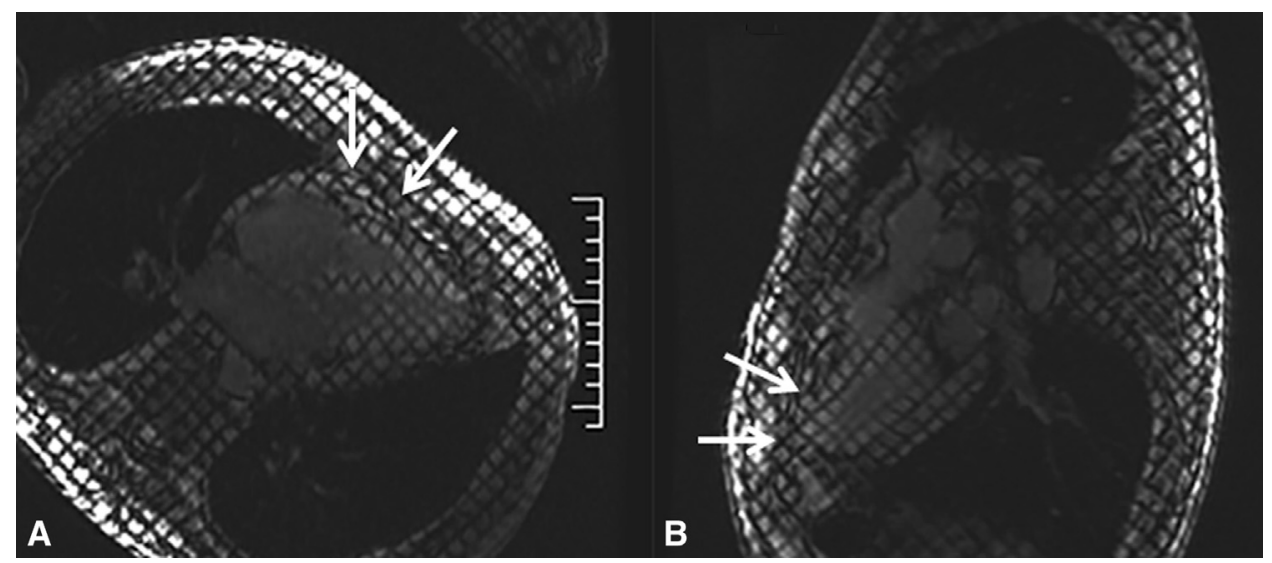

FIGURE 3. A and B, RF tissue tagging. The images demonstrate ventricular systole with constriction in normal-thickness pericardium in a $67-y e a r-o l d$ white woman with surgically confirmed constriction. 


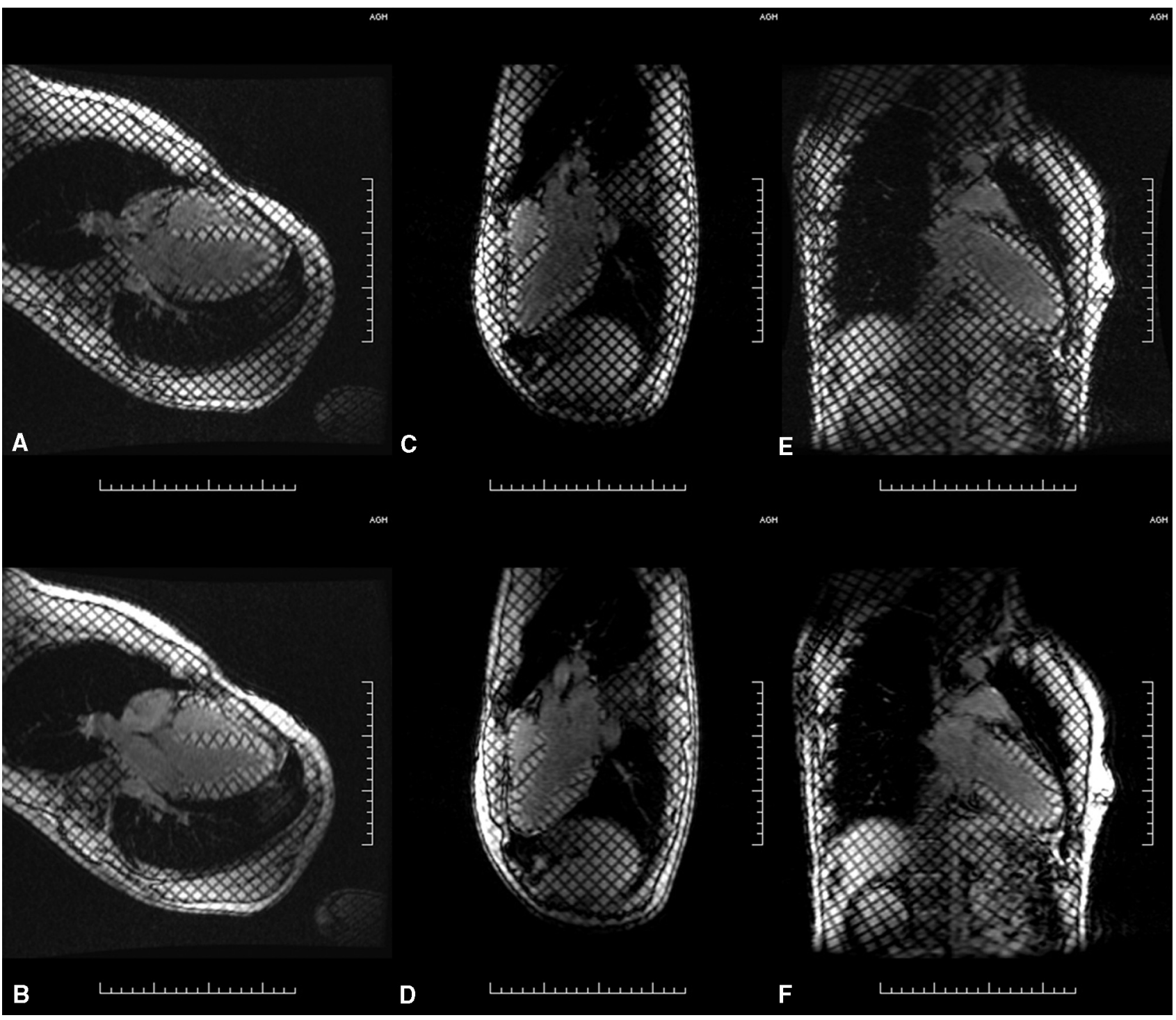

FIGURE 4. All 6 images depict a normal heart using RF tissue tagging. A and B, Standard 4-chamber view in ventricular diastole and systole, respectively, with complete slippage. C and D, A standard 3-chamber view in ventricular diastole and systole, respectively, with complete slippage. E and F, A standard 2-chamber view in ventricular diastole and systole, respectively, with complete slippage.

imaged. A selection bias naturally was present. The referred population was skewed toward symptoms because patients had been generally referred for a suspicion of CP using physical examination or other modalities to diagnose. Likewise, there was an inability to surgically confirm CP-negative patients; a negative diagnosis did not warrant surgery. As in all such "proof of concept" studies, surgery would not be performed simply to define sensitivity/ specificity because it is not ethically acceptable. That no patient crossed over to the surgical arm or had signs/ symptoms at 18-month follow-up of $\mathrm{CP}$ directly adds validity to our observations despite the lack of a surgical-comparator arm. During this study, only cardiac MRI and cardiac catheterization were compared; CT was not incorporated because of its inability to track physiologic pericardial interactions. Echocardiography was underrepresented, reflecting the underpowered ability to demonstrate clinical reliance for CP (except at specialized centers). ${ }^{5}$ There was also a lack of consistent use of non-CMR testing in all of the patients, reflecting individual physician references, because clinical conditions determined the noninvasive strategies. This lack of consistency generated inequity when comparing different modalities but did not negate the principal findings of CMR's ability to confirm the surgical findings. Late gadolinium enhancement was not performed in these studies, but we note it has been shown to predict the reversibility of $\mathrm{CP}$ after anti-inflammatory therapy. ${ }^{18} \mathrm{CMR}$ has certain inherent 
limitations: claustrophobia, difficulty with certain devices, and lack of uniform expertise in performing and interpreting results. Finally, this method of diagnosis is a learned interpretation and takes a certain amount of training to become proficient.

\section{CONCLUSIONS}

CMR via RF tissue tagging may offer a novel, accurate, noninvasive, and rapid manner in which to formally define $\mathrm{CP}$, as confirmed objectively at pericardiectomy surgery after the initial CMR diagnosis. CMR proved to be $100 \%$ accurate for the diagnosis of $\mathrm{CP}$, and no patient underwent an inappropriate pericardiectomy. Meanwhile, cardiac catheterization had an unacceptable number of falsepositives and false-negatives given the invasive nature of the test. It should be noted that this study was naturally unable to confirm the significant number of CP-negative patients. This "proof of concept" study suggests that, on further validation, CMR has the characteristics to be the new "gold standard" for CP as a stand-alone tool or while serving as a complementary modality when other modalities are equivocal.

\section{Conflict of Interest Statement}

Authors have nothing to disclose with regard to commercial support.

\section{References}

1. Wang ZJ, Reddy GP, Gotway MB, Yeh BM, Hetts SW, Higgins CB. CT and MR imaging of pericardial disease. Radiographics. 2003;23:167-80.

2. Nishimura RA. CP in the modern era: a diagnostic dilemma. Heart. 2001;86: 619-23.

3. Lieng LH, Oh JK, Schaff HV, Danielson GK, Mahoney DW, Seward JB, et al. CP in the modern era: evolving clinical spectrum and impact on outcome after pericariectomy. Circulation. 1999;100:1380-6.
4. Pichel IA, Concepción Fernández Cimadevilla O, de la Hera JM, Martín M. Letter to the Editor. Constrictive pericarditis: early diagnosis for better prognosis J Thorac Cardiovasc Surg. 2014;148:1766.

5. Khandaker MH, Espinosa RE, Nishimura RA, Sinak LJ, Hayes SN, Melduni RM, et al. Pericardial disease: diagnosis and management. Mayo Clin Proc. 2010;85:572-93.

6. Thavendiranathan $\mathrm{P}$, Verhaert D, Walls M, Bender JA, Rajagopalan S, Chung YC, et al. Simultaneous right and left heart real-time, free-breathing CMR flow quantification identifies constrictive physiology. JACC Cardiovasc Imaging. 2012;5:25-7.

7. Kovanlikaya A, Burke LP, Nelson MD, Wood J. Characterizing chronic pericarditis using steady-state free-precession cine MR imaging. Am J Roentgenol. 2001;179:475-6.

8. Biederman RW, Doyle M, Yamrozik J. Alternative use of cardiac MRI. In: Biederman RWW, ed. Cardiovascular MRI Tutorial: Lectures and Learning. Philadelphia, PA: Lippincott William \& Wilkins; 2008:196-200.

9. Axel L, Dougherty L. MR imaging of motion with spatial modulation of magnetization. Radiology. 1989;171:841-5.

10. Angheloiu G, Rayarao G, Williams R, Yamrozik J, Doyle M, Biederman RW Magnetic resonance characterization of septal bounce: findings of blood impact physiology. Int J Cardiovasc Imaging. 2015;31:105-13.

11. Wilson EB. Probable inference, the law of succession, and statistical inference. J Am Stat Assoc. 1927;22:209-12.

12. Kojima S, Yamada N, Goto Y. Diagnosis of CP by tagged cine magnetic resonance imaging. N Engl J Med. 1999;341:373-4.

13. Lachhab A, Doghmi N, Zouhairi A, Seghrouchni A, Wahid FA, Boulahya A, et al Use of magnetic resonance imaging in assessment of CP: a Moroccan center experience. Int Arch Med. 2011;4:36.

14. Troughton RW, Asher CR, Klein AL. Pericarditis. Lancet. 2004;363:717-27.

15. Bogabathina H, Biederman RW. Lack of slippage by cardiovascular magnetic resonance imaging is sine qua non for CP. Circulation. 2011;123:418-9.

16. Mikolich JR, Martin ET. CP diagnosed by cardiac magnetic resonance imaging in a pacemaker patient. Circulation. 2007;115:191-3.

17. Gopaldas R, Dao T, Caron N, Markley J. Predictors of in-hospital complications after pericardiectomy: a nationwide outcomes study. J Thorac Cardiovasc Surg. 2013; 145:1227-33.

18. Feng D, Glockner J, Kim K, Martinez M, Syed IS, Araoz P, et al. Cardiac magnetic resonance imaging pericardial late gadolinium enhancement and elevated inflammatory markers can predict the reversibility of $\mathrm{CP}$ after antiinflammatory medical therapy: a pilot study. Circulation. 2011;124:1830-7.

Key Words: cardiac magnetic resonance, cardiac surgery, constrictive pericarditis, magnetic resonance imaging, radiofrequency tissue tagging 